\title{
Les Jeunes et le Lien Social \\ De la Stigmatisation à la Compétence
}

\author{
Jacques Pain * \\ Alain Vulbeau**
}

\begin{abstract}
$\mathrm{R}$ revista Autrement, no final dos anos 70. «Juventude em ruptura: para lá da estigmatização, que mediações?», este era o título de um seminário do Congresso «Violências: da reflexão à ação». Mesmo se a trinta anos de distância, a fórmula soa como um refrão conhecido, as letras das canções já não são idênticas. Não se trata de uma simples repetição, mas da recorrência da questão dos jovens na sociedade. Para problematizar esta temática e as mudanças que aconteceram, é necessário interrogarmo-nos sobre diversos aspectos que irão ser desenvolvidos neste artigo: "os jovens e a violência dos jovens", as definições de conceitos como "juventude" e "incivilidade", a imagem da juventude através da estigmatização mediática, ou o papel da mediação de actores públicos como a polícia e as autarquias... Sem esquecer os jovens. Eles são, muitas vezes, reconhecidos como actores competentes.
\end{abstract}

Palavras-chave: violência urbana, práticas e discursos sobre a "incivilidade"; estigmatização e redefinição de competências do grupo jovem.

\section{La Violence: de quoi parlons-nous?}

La violence est un processus à géométrie variable (J.-C. Chesnay, 1981), complexe, toujours liée à des normes, à des conventions, à des situations. Nous savons ainsi tous que la violence varie - sa réalité et son appréciation, puisqu'elles vont ensemble - d'un pays à l'autre, mais encore d'une ville à l'autre, d'un tribunal à l'autre, en France même.

Les quartiers «sur» sensibles, «surspécifiques» (J. Pain, 2003), sont évidemment ceux où l'on trouve les établissements et les institutions dits sensibles, le plus souvent. Leur surspécificité violente tient au large panel de contre-indications sociologiques qui les identifient, en somme au cumul quantitatif et qualitatif des violences sociales, et à l'institutionnalisation d'un échec tendanciellement collectif (R. Castel, 2003).

Et c'est bien cette surspécificité qui fait la «violence urbaine» des deux tiers de ces quartiers: des incivilités, comme on dit désormais (dégradations, graffitis, nuisances), aux rébellions et aux violences «juvéniles», réactionnelles à l'institution et à l'autorité, et aux guets-apens ou aux émeutes anomiques, vécus parfois comme des «jeux» de guerre.

Bien sûr, la surspécificité, si elle est fait essentiellement par l'état des lieux, familial, social, culturel, scolaire, est nuée au cas par cas par la ville. Or, la «cité» est aux frontières centrales de la ville, enclave, ou péninsule, voire île ramassée sur sa territorialité. Un imaginaire insulaire, désespéré, agressif, désocialisé, dans des villes désertées par le travail.

La surspécificité de la cité, c'est qu'elle n'a rien de la ville «urbaine», que son destin lui vient de la colonisation intérieure. Et qu'on peut méditer sur l'internement de la pensée que recouvre l'antiphrase. Cité, civilité, civilisation, civisme, citoyenneté, du jour au lendemain les politiques retrouvent le chapelet républicain ou grec, de fait élitaire, et l'appliquent, en oubliant qu'ils transitent par leur antiphrase. L'incivisme, l'incivilité. Y aurait-il une incitoyenneté, une inCité? Une

\footnotetext{
* Professor de Ciências da Educação em Paris X Nanterre. Investigador n• Cref - Centre de Recherche en Educatiøn et Formation, Sector «Cris: école, terrains sensibles»na Université Paris X Nanterre. Contacto: pain.jacques@wanadoo.fr

* Sociólogo. Professor em Ciências da Educaçã• em Paris X Nanterre. Investigador no Cref - Centre de Recherche en Education et Formatiøn, Sector «Crise : école, terrains sensibles» na Université Paris X Nanterre. Contacto: alain.vulbeau@wanadoo.fr
} 


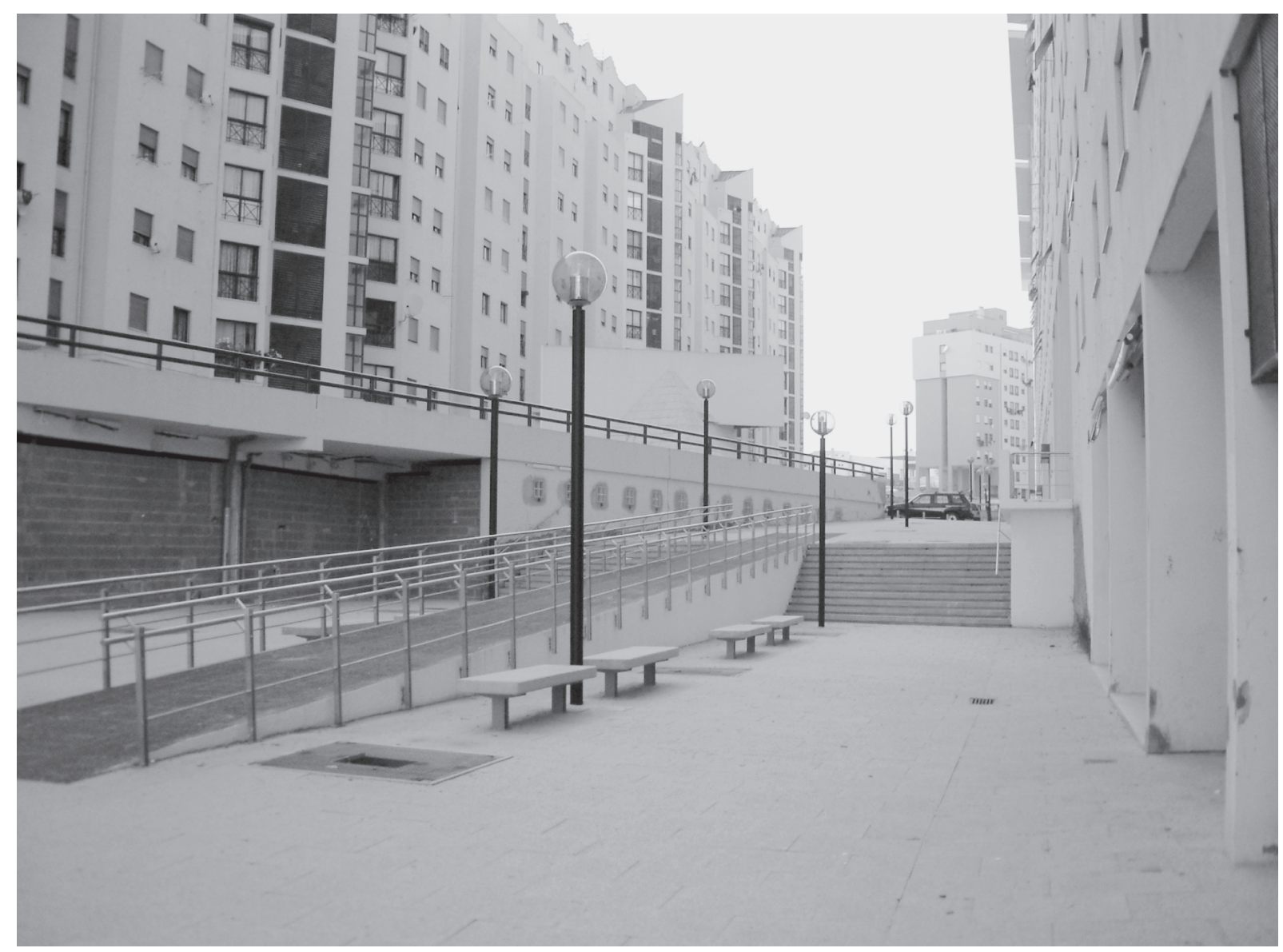

Foto de Dulce Moura

inurbanité incitée, qui friserait la désobéissance civile?

La citoyenneté peut alors n'être qu'une invocation, qui va de pair avec l'invocation d'une éducation à la loi, et si l'on n'y prête garde, la violence de la ville peut susciter davantage les moulins à prières et la magie d'intention que l'expérience collective d'une pédagogie du lien, concrète, ordinaire, quotidienne. Où la ville se construit comme un «espace éducatif élargi» (J.-M. Léon, 1992) dans le partage démocratique de l'institution.

\section{La Violence comme Conduite de Sécurité et Relation Sociale}

On pourrait avancer trois réflexions qui concourent pour faire de la violence une conduite plus fortement résultante de la conjonction sociale actuelle, et sinon à la banaliser, du moins à la rendre potentiellement ordinaire.

\section{Quelle Socialisation: des relations complémentaires aux relations para- doxales}

La socialisation est étroitement liée au premier entourage, à ses valeurs, et à son rapport aux institutions éducatives et aux appareils sociaux (L. Walgrave, 1992). Or, nous sommes en plein brouillage, et l'individuation se construit à présent dans le délitement, le désaccordement, le désagencement de ces appareils sociaux. D’un espace socioculturel plus ou moins «complémentaire», où s'articulent les institutions, illusoirement plein, plein d'emploi surtout, élitaire mais distributif, colonial mais rétributif, nous sommes passés à un espace socioculturel «paradoxal», halluciné par le vide social, trans-élitaire (je pense à l'overclasse désocialisée des golden boys américains) et duale, où domine une colonisation «médiane» (médiatique et indirecte) quasi culturelle. Chacun est renvoyé à soi, à son destin, dont rien ni personne ne peut plus assurer qu'il fasse le compte avant long terme. 


\section{La Société comme Matrice de Déve- loppement}

Ce sont là des matrices sociétales à dimension symbolique, que l'on peut sommairement entendre comme des agencements de conduite virtuelle, comme des idéologies locales, qui vont constituer une modélisation, une mentalisation, où s'exerceront les itinéraires de socialisation, d'individuation. Il y a là toute la question de l'enveloppe sociétale, du contenant social, dont la sociologie ne nous livre que les composantes, et rarement l'effet psychosociologique ou microsociologique sur le terrain. Or, s'il est vrai que les banlieues sont très différentes, très contrastées, que les quartiers qui les forment sont des mondes humains à part entière et qu'il faut sortir des stéréotypes et de la stigmatisation, il n'en reste pas moins que l'ambiance, l'atmosphère, le climat, induisent idéologiquement - les médias aidant - un état de la relation sociale largement phagocyté par l'angoisse.

La vie quotidienne est hyper complexe, mais on la verra dès lors comme l'imbrication de situations plus ou moins «infestées» par l'angoisse, où les conduites et les comportements sont des réponses quasi contraintes plutôt de soumission, de fuite ou de lutte (H. Laborit, 1994) c'est-à-dire d'évitement, de cristallisation, de métabolisation, de l'angoisse «individuale». La violence sera, à ce stade intersubjectif, tout d'abord le refus ou l'impossibilité de faire de ces réponses une communication normativement socialisée, ensuite un acting on moteur (J. Selosse, 1997) visant le réel et autrui, dans une réaction plus ou moins construite de protection, de sécurité, une capture princeps de l'autre éludant la rencontre (J. Pain, 1998).

\section{Désidentification et Humanisation Violente}

Nous admettrons, par conséquent, que l'humanisation est en proie à une culture de la violence, socialisante, sociétale, plurisécuritaire, où les schémas d'aliénation identitaire traditionnellement structurants sont attaqués en leur fondement. Comment tenir à ce qui ne vous tient pas? On pourrait comprendre alors que ce puzzle brouillé soit une matrice de «désidentification» (J. Selosse, 1997) relative, particulièrement pour les dix-quatorze ans, qui nous occupent un peu plus chaque jour. Une «désidentification» narcissique typiquement individualiste. Comme s'ils tentaient

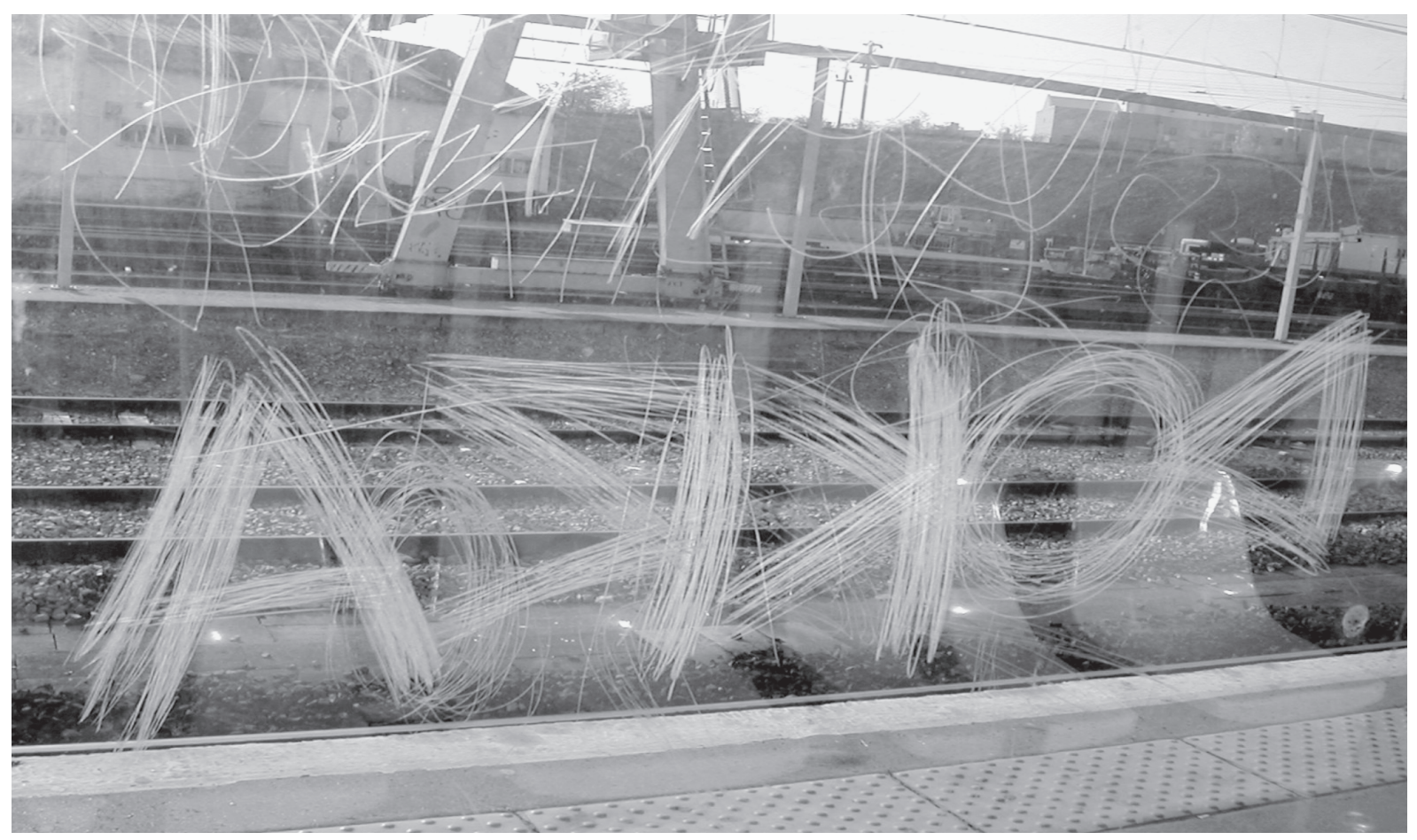

Foto de Dulce Moura 
d'éradiquer le lien institutionnel, dans une lutte à mort avec leur essence sociale. Ni futur, ni identité, ni loi ni règle communes. La convention est forclose. La négativité l'emporte.

«Le contrôle social est effectif quand la socialisation intègre les règles sociales qui régulent les comportements individuels. Le contrôle social est inadéquat quand la socialisation et les règles sociales sont désorganisées.»(J. Selosse, 1997).

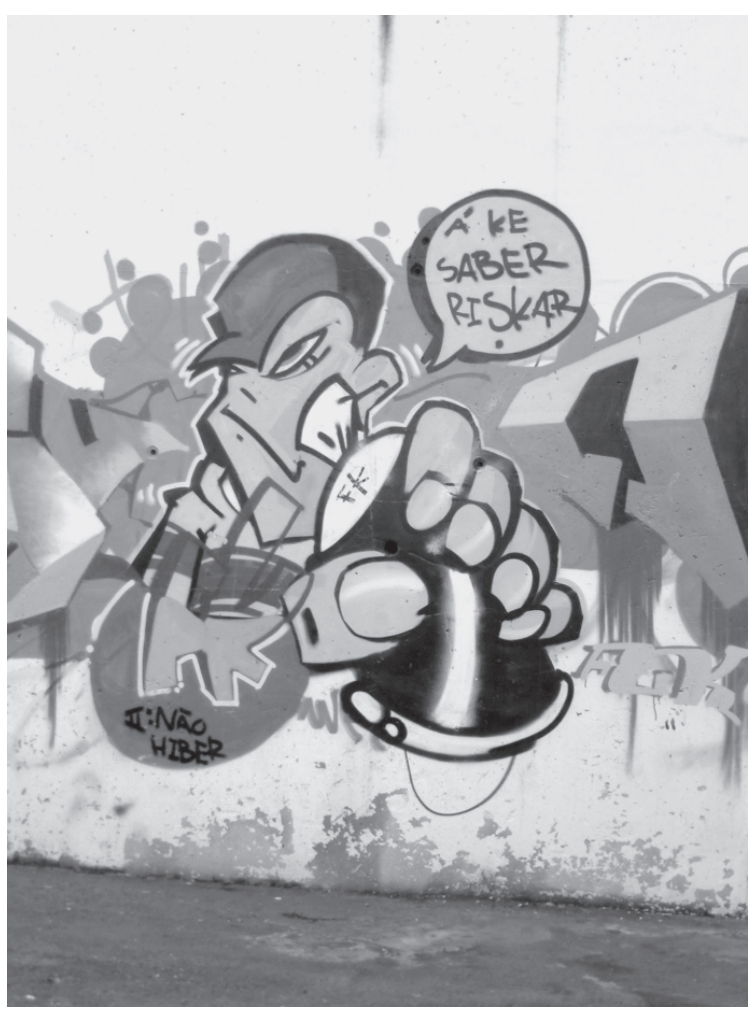

Foto de Dulce Moura

\section{Théorie Fondée et Socio-Ethnographie}

Afin de s'inscrire pleinement dans un registre susceptible d'articuler la réflexion à l'intervention, il est nécessaire non seulement d'associer mais de faire interagir théorisation et analyse des pratiques. On va d'abord l'envisager de façon générale avant de l'appliquer plus spécifiquement à la question des «jeunes en rupture».

Le concept de «théorie fondée», de Barney Glaser et Anselm Strauss (1995), est une façon de questionner cette interaction en privilégiant deux aspects. D'abord, cela permet de placer au centre de l'analyse des situations qui sont des concentrés d'espace-temps où se jouent localement des processus globaux. Ensuite, ce concept permet de viser moins une perspective de vérification d'une théorie a priori, que dans celle d'une élaboration provisoire et d'une théorisation à moyenne portée.

Une autre façon d'interroger cette interaction est de se situer dans une perspective de recherche fondée sur des démarches de terrain, attentives aux singularités locales. Il n'est pas possible ici de faire l'inventaire de toutes ces démarches (recherche-action, intervention institutionnelle, analyse clinique des pratiques) mais, en insistant sur l'une d'entre elles, il s'agira moins de limiter le débat que de donner un exemple de prise en compte de cette perspective. La démarche socio-ethnographique s'appuie sur trois points fondamentaux: la reconnaissance d'une pluralité de mondes sociaux d'échelles différentes, traversés par des processus d'appropriation et de dispersion, de conflits et d'échanges; la volonté de procéder à une approche compréhensive des acteurs, et donc de ne jamais se contenter du «discours sur», qui est souvent à la base des processus d'étiquetage et de stigmatisation; la prise en compte d'une pluralité de sources d'information, qu'un vécu prolongé avec les acteurs concernés permet de mobiliser. Sur le thème des jeunes, plusieurs recherches ont produit des résultats appuyés sur tout ou partie des points cités plus haut. On peut citer, parmi d'autres, les enquêtes de Pascal Duret sur les grands frères, de David Lepoutre sur la vie dans un grand quartier d'habitat social, de Béatrice Sberna sur le rap à Marseille, et les travaux de Catherine Neveu et Michel Kokoreff sur les relations des jeunes avec les institutions locales.

\section{Définitions et Indéfinitions de la Jeunesse}

Parmi les points de réflexion, il serait bon de s'interroger sur les notions de définition et d'indéfinition de la jeunesse. En effet, les travaux maintenant classiques de la sociologie de la jeunesse d'Olivier Galland (1997) et de Gérard Mauger (1994), ont montré l'existence et la coexistence de définitions différentes de la jeunesse et des jeunes, engageant des points de vue théoriques et pratiques très contrastés. La jeunesse peut apparaître comme une catégorie d'âge, définie par les usages d'une intervention institutionnelle (ce sont les 12/17 ans, les 16/25 ans, les 18/30 ans, etc.). On peut aussi en faire un âge de la vie, 
centré sur une fonction principale comme l'imitation ou l'expérimentation. Enfin, on peut y lire un rapport de génération, où l'on fait partie d'une génération de jeunes située dans une sensibilité distincte de la génération «adulte».

Ces définitions s'entendent sur un point, l'existence de la jeunesse existe, mais diffèrent sur d'autres points, ce qui amène à prêter la qualité de jeune à des individus très différents. Il y a aussi à articuler les points de vue d'indéfinition sur la jeunesse. Ainsi, celui, mentionné par Bourdieu (1980), qui fait de la jeunesse, une notion idéologique, dont l'enjeu est de masquer les rapports sociaux de classe. On sait aussi comment le terme «jeunesse» sert à désigner certaines populations, en particulier les jeunes hommes des minorités maghrébines vivant dans les quartiers d'habitat social.

En fait, et ce pourrait être un premier point du débat, la pluralité des définitions et des indéfinitions, tient peut-être à la pluralité des acteurs et des intervenants, et, en définitive, à l'éclatement du champ de la jeunesse au plan des politiques publiques. Il faut tenir compte de cette pluralité, qui est moins le révélateur d'une confusion théorique que la conséquence de certaines formes d'actions publiques, notamment dans le cadre de la politique de la ville. Cela suppose alors de s'interroger sur le partenariat et sur l'attribution respective des définitions de la jeunesse, aux acteurs concernés. Il faut ajouter que les jeunes eux-mêmes participent à la construction de cette définition/ indéfinition.

\section{Stigmatisation et Médias Télévisés}

Aborder la question de la stigmatisation est en rapport avec les formes qu'elle prend et avec les conséquences qu'elle engendre. Pour ce qui est des formes, il est possible de questionner les figures typées par les médias. Ainsi, les journaux

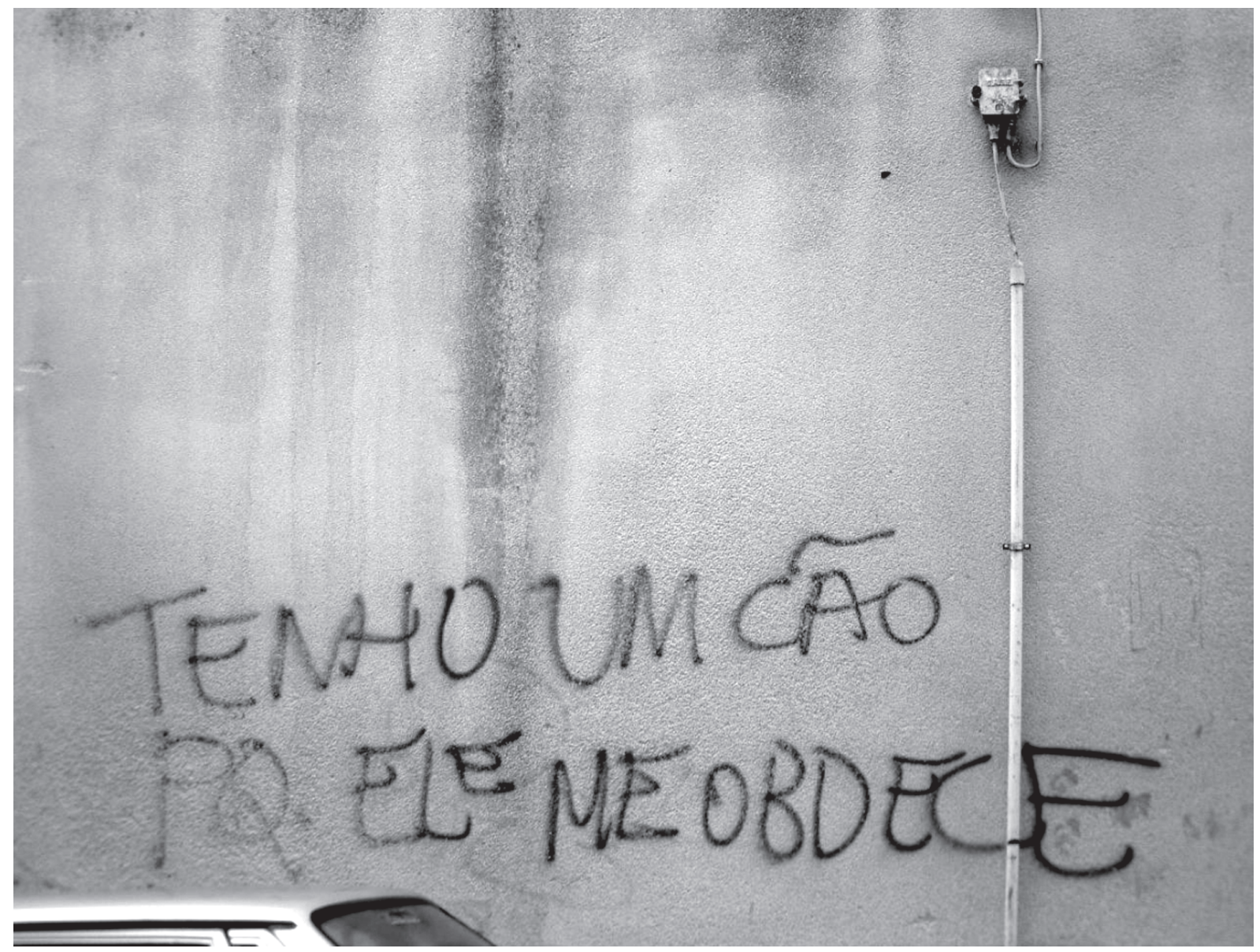

Foto de Dulce Moura 
télévisés ont donné pendant de longs mois des images des «jeunes en rupture» à travers des reportages très nombreux. Divers types de mises en scène sont employés:

Les «plans rapprochés», où les visages sont «floutés» ou «mosaïqués», et les voix rendues méconnaissables par des effets de sampling;

Les séquences «à distance», prises depuis les véhicules des forces de l'ordre engagées dans leur tournée, et leurs confrontations quotidiennes plus ou moins violentes.

Il est important de s'interroger sur les modes de scénarisation, les types de sollicitation de la parole, les arguments et les discours des personnes interviewées, etc. Il faut aussi envisager les effets d'ambiance de ces images dans le contexte sécuritaire de ces dernières années. Un point fondamental pour nous est la déshumanisation potentielle de ces procédés: les jeunes qui n'ont plus de visages ni de voix humaines, sont vus de loin comme les animaux sauvages de cette quasi-réserve naturelle que serait la «zone de non-droit».

\section{Les Rôles de la Police}

Les policiers forment une catégorie d'acteurs incontournables de la dernière décennie. L'intervention policière s'est affirmée comme une mission de plus en plus diversifiée, incluant, au-delà de la traditionnelle fonction répressive, des compétences dans les secteurs de la prévention, de la protection, de l'animation, de l'insertion professionnelle et sociale, voire également dans l'expertise en matière de connaissance sur les jeunes. Autrement dit, la police apparaît à travers des images aussi bien coercitives, liées à ce que l'on appelait au dix-neuvième siècle la «correction paternelle», que des images protectrices voire maternantes. Captant presque tous les rôles sociaux, la police s'installe dans ce que nous appelons, en reprenant G. Mendel, un imaginaire d'autorité psychofamilial.

Cette diversification, qui correspond à une politique de mise en øuvre de la police urbaine de proximité dans les années 90 , est actuellement dans une phase de remise en cause explicite. On peut s'interroger sur les effets de brouillage liés à la diversification de cette action policière (notamment du côté de la médiation), et à ceux qui peuvent découler des récents changements de tendance de cette action.

\section{Le Rendement Discursif des «Incivilités»}

Du côté des discours, il faut s'intéresser à la spécificité des langages déployés dans les champs concernés, en particulier en identifiant des mots clés ou des mots valises, dont le rendement laisse songeur. Ainsi, le terme «incivilité», qui a fait une carrière remarquable, désigne, d'une part, ce qui n'est pas poursuivi tout en étant ouvertement pénalisable, et, d'autre part, ce qui n'est pas qualifiable pénalement mais qui apporte un trouble social suffisant pour mériter d'entrer dans la catégorie des infractions réelles. Il est fréquent d'entendre dire que les incivilités «pourrissent la vie», ce qui est une manière, sur le plan des métaphores, d'enclencher une prolifération d'images stigmatisantes sur la putréfaction de certains groupes sociaux et sur une hygiène de défense sociale appropriée.

La fonction des usages du mot «incivilité» est double. Ce terme permet de créer un continuum entre les domaines de l'infra-pénal et du pénal; autrement dit, un crachat ou un regard, voire une grimace, peuvent passer d'une absence de «savoir vivre» à la menace potentielle. Ce mot donne une exclusivité de regard et d'interprétation à un processus de pénalisation du social. Ce processus n'est pas nouveau puisque Michel Foucault avait montré qu'il avait servi, au dix-neuvième siècle, de support à la création de maisons de correction, dont le public cible était des jeunes pas assez coupables pour aller en prison mais pas assez innocents pour être remis en liberté.

\section{De la Stigmatisation à la Compétence}

Les approches socio-ethnographiques sur la vie des jeunes dans les quartiers d'habitat social ont mis en évidence la notion de compétence juvénile, à travers notamment l'usage des réseaux d'interconnaissance, la capacité de négociation avec les institutions locales, la fluidité des parcours sur des territoires d'échelles différentes. Les pratiques liées à la culture hip hop illustrent bien également la question de la compétence des jeunes. Ceux-ci, à partir de leurs sociabilités propres, localisées dans des lieux souvent stigmatisés, génèrent des activités, des ateliers, des expositions, des spectacles, et des profils d'intervenants dans les secteurs sociaux et culturels. 


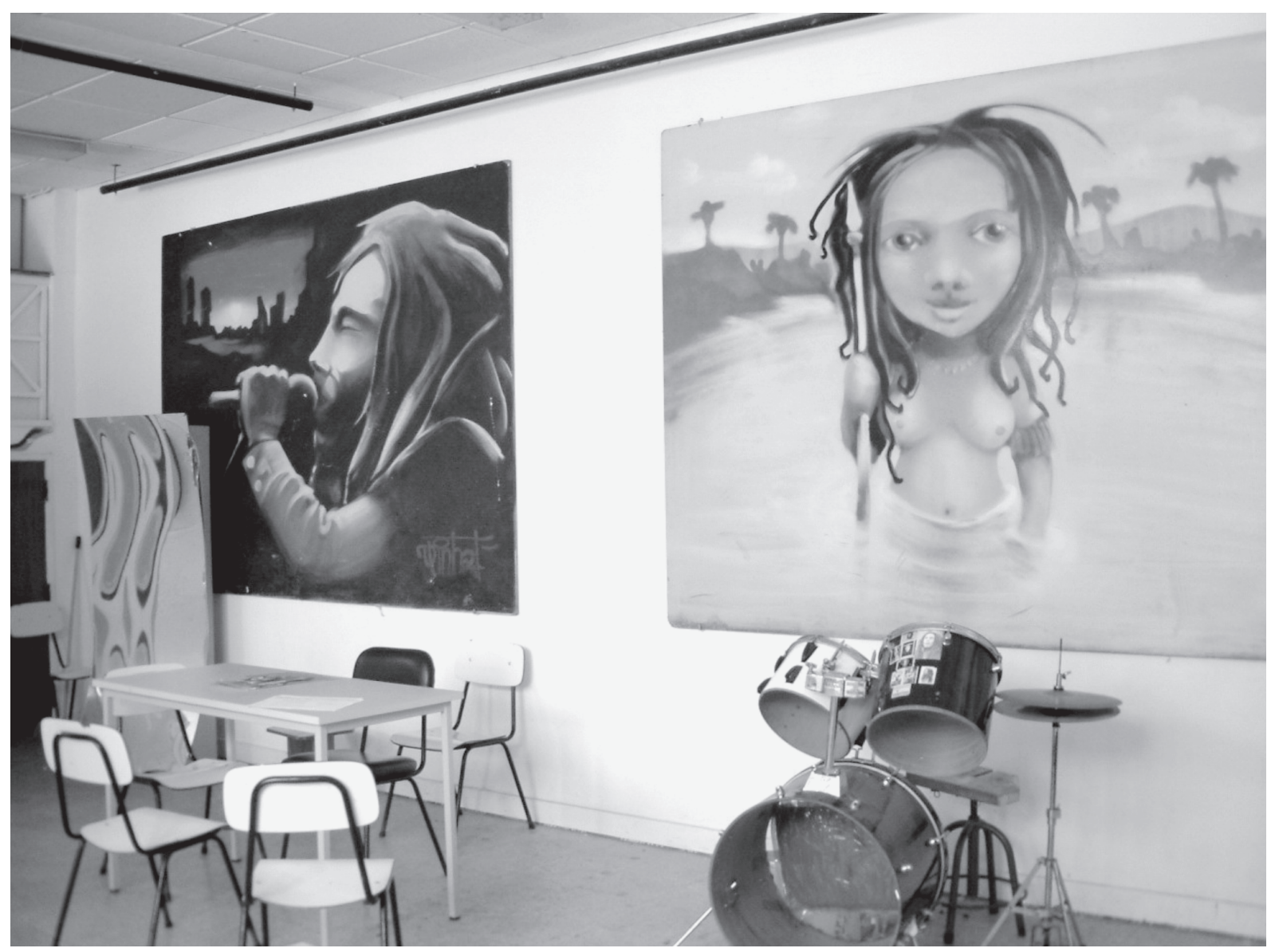

Foto de Dulce Moura, Programa Escolhas, Vale da Amoreira

La connaissance fine de situations locales permet de voir les incidences souvent bénéfiques de la transformation de ces sociabilités dans les parcours d'insertion de certains jeunes, et ce, surtout quand il existe sur place des modes de consultation et de participation renouvelant les débats civiques.

Ces processus de conversion des sociabilités et de participation citoyenne relèvent de la socialisation secondaire et des relations de réciprocité entre les générations. Dans une perspective de médiation et de production des «métiers de la ville», ces processus présentent deux sortes d'effets: d'une part, certains objets de la stigmatisation se retournent en signes de créativité, de développement local et de leviers d'intégration; d'autre part, les acteurs spécifiques de ce champ occupent «spontanément» le chaînon manquant dans les liens intergénérationnels, lien que les institutions s'épuisent, souvent sans succès, à mettre en œuvre.

\section{Les paradoxes de la citoyenneté et le retour à la loi: comme norme ou comme valeur?}

Nous terminerons par trois nouvelles réflexions centrées sur la possibilité d'une réémergence de la citoyenneté, dans le contexte désocialisant, désidentifiant, violent, des villes, et des jeunes dits difficiles, d'aujourd'hui.

\section{La Citoyenneté comme Effet Social de Situations}

Quelle citoyenneté? Pour quoi faire? On n'enseigne pas plus la citoyenneté que le civisme, et l'inspection générale soutient depuis longtemps l'idée que l'éducation civique n'est pas une discipline, un corpus ou un mémento formel, mais une pratique en situation. Ce qui est vrai pour l'école l'est d'autant plus pour la ville. Or, on voit fleurir des initiatives qui laissent rêveur, des actions- 
-spectacles pour la citoyenneté, des journées du citoyen, des semaines républicaines, on nous annonce des cercles de citoyens en région parisienne. Bien sûr, si l'État, les médias, la SNCF, la Poste, la RATP, les municipalités des villes sensibles, conjoignent leurs efforts, on peut espérer une influence, à l'égal peut-être des grandes campagnes norvégiennes contre les violences à l'école (Dan Olweus), mais on sait que l'influence n'atteint son acmé qu'en touchant la vie concrète des personnes. C'est toute la limite aussi bien du retour à la loi, injonction judiciaire fondée en droit, qui pourrait autant, à tout moment, basculer dans un retour trivial et normatif de la loi, sans l’éducation et la prévention précoces qui «conscientisent» à la loi.

\section{Respect et Éthique du lien}

En fait, on privilégie la morale, une morale de l'institution, reconvoquée, pour la forme, dans l'urgence urbaine. Celle justement qui exaspère les adolescents et les jeunes. Ainsi, par exemple, de ce discours commun sur les familles des banlieues, leurs carences, leur impuissance, que l'on entend au cœur même des institutions d'état. Quand on s'en donne les moyens, en allant les chercher, ces familles, bon nombre d'entre elles réagissent. Mais le contrat social est à refaire, il s'agit de «refaire institution», comme on dirait refaire surface dans la cité. Reprendre au degré zéro, déviance, délinquance, violence, discerne, différencier, expliciter. Prendre les choses au sérieux, dès la petite enfance, prendre la famille, la rue, l'école, au sérieux, pour la vie. C'est en construisant des réseaux, des constellations, d'intérêts, de relation, scellés par le «respect», au jour le jour, que le sujet retrouve une éthique, car il se structure alors lui-même comme sujet de l'institution, instituant «vrai» d'un collectif social humain. C'est dans l'institutionnalisation expérientielle ouverte, dans l'expérimentation sociale, du respect, que le sujet teste la société, et intègre, par des signes, par des actes, par des situations partagées, le sens d'une éthique du lien. Dans la famille, dans la rue, à l'école.

\section{Vers une Pédagogie de la Ville}

Grandir, pari d'institution? Autoriser et s'autoriser à grandir ! La ville est bien cet ensemble éducatif élargi, où la citoyenneté est un effet associatif, d'un collectif qui transcende les communautés et les «entreprises citoyennes». Où, dès lors, il y a à faire une pédagogie institutionnelle du lien, si ce n'est dans la famille, alors à la crèche, à l'école, dans le quartier, mais très tôt, très vite. Une pédagogie du pouvoir social (G. Mendel), qui articule la loi, la sanction, le respect, la responsabilité, comme des valeurs et non plus seulement comme des normes. C'est tout l'enjeu de la «vie scolaire», à l'école, en maternelle, en primaire, en secondaire. Tout l'enjeu d'une démocratie scolaire authentique et conseilliste. Car la vie est aussi un langage à faire en commun, avec des lieux, des limites, des lois, de l'interdit, des partenaires. Ça commence par Vivre ensemble impossible d'y échapper; sans se Traiter - de tout, ou plutôt de rien; dans une certaine Paix sociale - consentie par nécessité vitale. Les enfants comprennent ça très bien, à l'école. Rien de miraculeux. De l'obstination, de la parole, du rituel, de la décision, du groupe. Car l'individu négatif, tout comme l'individu positif (R. Castel, 2003), est en dernière extrémité dans la dépendance de l'autre. C'est pour ça que l'éducation à la loi et à la citoyenneté sont des questions de pédagogie!

«La cognition sociale s'apprend et devrait être enseignée au même titre que les autres domaines de cognition: mathématiques ou physiques.» (J. Selosse, 1997).

\section{Action Publique, Jeunes et Médiation}

Pour conclure, il ne faudrait pas penser que les sociabilités des jeunes deviennent aussi facilement des supports d'intervention et de médiation. En amont de leur prise en compte institutionnelle, les logiques de pénalisation du social forment un barrage efficace. En aval, des activités comme le hip hop ou la production de médias locaux, sont rarement des solutions définitives mais des problèmes supplémentaires à gérer finement. Ces derniers révèlent les capacités des institutions à prendre en compte certaines dimensions relationnelles de l'action publique comme, entre autres, le soutien politique des services administratifs, la valorisation de la transversalité au service des projets, la mise à disposition, pour les acteurs locaux, de 
compétences et de moyens techniques, la capacité à détecter et traiter les conflits, la publicité de l'évaluation des actions, etc.

Pourtant même (et surtout), si ces interactions sont porteuses de conflits, c'est à partir de ces processus que s'exprimeront l'intelligence politique vis-à-vis des banlieues, les bonnes volontés de la médiation, et les contrariétés inhérentes à la mise en œuvre d'une cohésion sociale fondée sur la réciprocité.

\section{Bibliographie}

BEAUD, S, 1997, "Postface pour une ethnographie sociologique", in BEAUD, S. Weber, F., Guide de l'enquête de terrain, Paris, La Découverte.

BONNEMAIN, C., 2000, "Les incivilités: usages d'une nouvelle catégorie", in BAILLEAU, F., Gorgeon, C., Prévention et sécurité: vers un nouvel ordre social, Saint-Denis, DIV.

BOUCHER, M., VULBEAU, A., 2003, Émergences culturelles et jeunesse populaire: turbulences ou médiation?, Débats Jeunesse, L'Harmattan (à paraître).

BOURDIEU, P., 1980, "La jeunesse n'est qu'un mot", Questions de sociologie, Paris, Minuit.

CASTEL, R., 2003, L'Insécurité sociale, qu'est-ce qu'être protégé, Paris, Seuil.

CHESNAIS, J. C., 1981, Histoire de la violence, Paris, Laffont.

DURET, P., 1996, Anthropologie de la fraternité dans les cités, Paris, PUF.

CNAF, 2001, "Police et social", Informations sociales, n. ${ }^{\circ} 92$.

FOUCAULT, M., 1975, Surveiller et punir, Paris, Gallimard.

GALLAND, O., 1997, Sociologie de la jeunesse, Paris, Armand Colin.

GLASER, B., STRAUSS, A., 1995, "La production de la théorie à partir des données", Enquête, n. ${ }^{\circ} 1$.

KOKOREFF, M., 2003, La force des quartiers, Paris, Payot.

LABORIT, H., 1994, La légende des comportemnts, Paris, Flammarion.

LABORIT, H., 1976, Éloge de la fuite, Paris, Laffont.

LABORIT, H., 1970, L'agressivité détournée, Paris, UGE.

LÉON, J. M. cf. PAIN, J., 1992, Ecoles: violence ou pédagogie?, Vigneux, Matrice.

LEPOUTRE, D., 1997, Cour de banlieue: codes, rites et langages, Paris, Odile Jacob.
«Les sujets qui grandissent dans un univers de force, dans un univers de violence, mais aussi dans un univers de laisser-faire et d'indifférence, n'apprennent pas à canaliser leur agressivité et à la socialiser. Ils sont dans un univers de type réactionnel, au niveau réflexe le plus élémentaire, et ils continuent à fonctionner sur ce mode réflexe. C'est pourquoi une des dimensions fondamentales de l'éducation c'est de pouvoir, entre le désir et la pulsion, introduire la médiation de l'autre.» (J. Selose, 1997).

MANÇO, A., AMORANITIS, S. (dir.), 1999, Délégation par abandon, Les Politiques Sociales, n. ${ }^{\circ}$ 3-4.

MAUGER, G., 1994, Les jeunes en France: état des recherches, Paris, La Documentation Française.

MOZÈRE, L., PERALDI, M., Rey, H., 1999, Intelligence des banlieues, La Tour d'Aigues, L'Aube.

NEVEU, C., 2001, "L'accès à l'espace public entre politiques publiques et "dérive ethnique". L'expérience d'associations de jeunes à Roubaix", in Vulbeau, A. (dir.), 2001, La jeunesse comme ressource : expériences et expérimentations dans l'espace public, Ramonville-Saint-Agne, Obvies/Érès.

OLWÉUS, D., 1999, Violence, harcélement et brutalités entre élèves, Paris, ESF.

PAIN, J., 1998, La non violence par la violence (une voie difficile), Vigneux, Matrice.

PAIN, J., 2003, La Société commence à l'école, Vigneux, Matrice.

PAIN, J., 2003, Pour des pédagogies actives, avec ou contre l'école, Vigneux, Matrice.

PAIN, J., VULBEAU, A., 2003, L'Invention de l'autorité, Vigneux, Matrice.

SBERNA, B., 2001, Une sociologie du rap à Marseille: identité marginale et immigrée, Paris, L'Harmattan.

SELOSSE, J., 1997, Adolescence, violences et déviances, Vigneux, Matrice.

STRAUSS, A., 1992, La Trame de la négociation: sociologie qualitative et interactionnisme, Paris, L'Harmattan (cf. le chapitre "Une perspective en terme de monde social").

VULBEAU, A. (2002), «Le langage de l'insécurité: regard sur le journal télévisé», Claris, le bulletin n. ${ }^{\circ} 2$, édition électronique.

WALGRAVE, L. (1992), Délinquance systématisée des jeunes et vulnérabilité sociétale, essai d'une théorie intégrative, Paris, Méridiens Klincksieck. 